\title{
Wideband Dual Sphere Detector of Gravitational Waves
}

\author{
M. Cerdonio, ${ }^{1}$ L. Conti, ${ }^{1}{ }^{*}$ J. A. Lobo,${ }^{2}$ A. Ortolan, ${ }^{3}$ L. Taffarello, ${ }^{4}$ and J. P. Zendri ${ }^{4}$ \\ ${ }^{1}$ INFN Padova Section and Department of Physics, University of Padova, via Marzolo 8, I-35100 Padova, Italy \\ ${ }^{2}$ Departament de Física Fonamental, Universitat de Barcelona, Diagonal 647, 08028 Barcelona, Spain \\ ${ }^{3}$ INFN, Laboratori Nazionali di Legnaro, via Romea 4, I-35020 Legnaro, Padova, Italy \\ ${ }^{4}$ INFN Padova Section, via Marzolo 8, I-35100 Padova, Italy \\ (Received 2 November 2000; revised manuscript received 16 March 2001; published 28 June 2001)
}

\begin{abstract}
We present the concept of a sensitive and broadband resonant mass gravitational wave detector. A massive sphere is suspended inside a second hollow one. Short, high-finesse Fabry-Perot optical cavities read out the differential displacements of the two spheres as their quadrupole modes are excited. At cryogenic temperatures, one approaches the standard quantum limit for broadband operation with reasonable choices for the cavity finesses and the intracavity light power. A molybdenum detector, of overall size of $2 \mathrm{~m}$, would reach spectral strain sensitivities of $2 \times 10^{-23} \mathrm{~Hz}^{-1 / 2}$ between 1000 and $3000 \mathrm{~Hz}$.
\end{abstract}

DOI: 10.1103/PhysRevLett.87.031101

PACS numbers: 04.80.Nn, 95.55.Ym

Resonant mass detectors of gravitational waves (GW) are commonly indicated as narrow band devices. In currently operating cylindrical bar detectors [1], all equipped with resonant transducers, the bandwidth, even in the limit of quantum limited performance of the final displacement readout, would not open up for more than about the frequency interval between the resulting two mechanical modes of resonance, currently about $30 \mathrm{~Hz}$.

This stems, however, from the noise performance of the employed readout systems. A general analysis of the problem, valid for any linear detector, has been given in Ref. [2]. Secondary resonant masses are linked to the main resonant mass to efficiently couple the signal amplitude to the final readout, but then the bandwidth is limited to a fraction of the main resonator frequency. Such a coupling is poorer the smaller the total number $n$ of resonators, and, correspondingly, the bandwidth decreases with $n$. To open the bandwidth, one would have to use multimode systems [3,4] with $n \geq 3$, but, until now, only two-(mechanical) mode systems have worked their way into operating detectors, giving a fractional bandwidth $\Delta f / f \ll 0.1$.

However, if a single mechanical resonator were driven only by its thermal noise and by a signal force, the signal to noise ratio would be independent of frequency, and thus the band would open up provided enough signal amplitude can be coupled to the final readout. The possibilities offered nowadays by optomechanical systems are such that the interplay between the back-action of the radiation pressure and the photon counting noise in a high finesse, high power Fabry-Perot cavity would allow enough signal coupling [5] to get broadband operation at the Standard Quantum Limit (SQL) (see below).

We have been attracted by the possibilities offered by optical readout systems, as vigorously developed for interferometric GW detectors, and more recently applied in connection with cryogenic bar GW detectors [6,7]. We take a Fabry-Perot optical cavity as the motion sensor. In a system under development [7], the length of the sensor cavity is compared to that of a second cavity, separately kept, which acts as a reference. We do not take into account here the noise introduced by the reference cavity, assuming for simplicity that it is negligible. With a sensor cavity length of the order of centimeters, there is no loss of signal strength for finesses $F$ as high as the highest attainable with current technology, $F=10^{6}$, for $\mathrm{GW}$ in the $\mathrm{kHz}$ range. So we have considerable freedom to vary the finesse and the light power $P$ incident on the cavity, in search for optimal conditions at a chosen frequency, which do not demand unreasonable values for these parameters.

Let us then turn to the primary mechanical resonator, whose motion is directly related to the incoming GW. We take into consideration both solid and hollow spheres as resonant systems of interest. They are very attractive for a number of reasons, and in fact they have received significant attention in the literature of the past few years [8-11]. Spherical detectors are omnidirectional, have a more efficient coupling to the GW field relative to cylindrical bars, both in the first and in the second quadrupole mode, and enable a deconvolution of the GW signal if they are equipped with five (or more) suitable motion sensors $[8,9,12]$. For instance, a chirp signal from a merging compact binary can be fully deconvolved with a spherical detector [13]. Two spheres would make up for a complete observatory, in which all parameters characterizing the incoming wave (e.g., velocity, direction, polarization) can be resolved [14,15] (see also [16]). Located close to an interferometric detector, a spherical detector could be used for searches of stochastic background [17]. Such capabilities make of the spherical detector a conceptually unique device.

However, the sensitive spherical detectors proposed in the past suffer from the above discussed bandwidth limitation. As an example, a hollow sphere of $\mathrm{Cu}_{0.9} \mathrm{Al}_{0.1}$, $4 \mathrm{~m}$ in diameter and $0.3 \mathrm{~m}$ thick, cooled at sub-Kelvin temperatures and equipped with resonant transducers and a quantum limited readout, gives a spectral strain noise as 
low as $6 \times 10^{-24} \mathrm{~Hz}^{-1 / 2}$ [11], but only in two bands of 35 and $135 \mathrm{~Hz}$, respectively, around the first and second quadrupole resonances at 350 and at $1350 \mathrm{~Hz}$.

Let us then consider a spherical detector with nonresonant optical readout. We need to integrate the two mirrors of each Fabry-Perot sensing cavity in two separate systems, which must be cold, massive, and of high mechanical $Q$ factor; otherwise the thermal noise would be unacceptably large. We are thus led to the concept of a GW detector based on a massive dual sphere system of resonators: a hollow sphere which encloses a smaller solid sphere (see Fig. 1). Motion sensors in this system will be optical Fabry-Perot cavities formed by mirrors coated face to face to the inner surface of the hollow sphere and to the solid sphere, in either of the two configurations proposed, respectively, in Refs. $[8,12]$.

The main sources of noise are thermal noise in the large detector masses, back-action noise introduced by the radiation pressure, and photon counting noise. Given the optical figures, the evaluation of all three contributions for our design is straightforward, as the spectrum of the resonant frequencies of the two spheres is known $[10,11]$ once their material(s) and dimensions are fixed.

Assuming the same material is used for both spheres, and that the inner one of radius $a$ fills up almost completely the interior of the other (external radius $R$, internal radius $\gtrsim a$ ), the first quadrupole resonance of the outer hollow sphere is at the lowest frequency, while that of the inner solid sphere is $2-3$ times higher. The frequency region in between is of particular interest: the GW signal drives the hollow sphere above resonance and the solid sphere below resonance. The responses of the two resonators are then out of phase by $\pi$ radians and therefore the differential motion, read by the optical sensors, results in a signal enhancement. In this region only a small number of nonquadrupole resonances occur, which are not GW active. The pattern repeats for the two second quadrupole modes at higher frequency and so on. At a few specific frequencies above the first quadrupole resonance of the solid sphere, under the combined effect of the response to GW of all the quadrupole modes, their responses subtract and the sensitivity is reduced and eventually lost in a few narrow bands. In this higher frequency region, in addition to

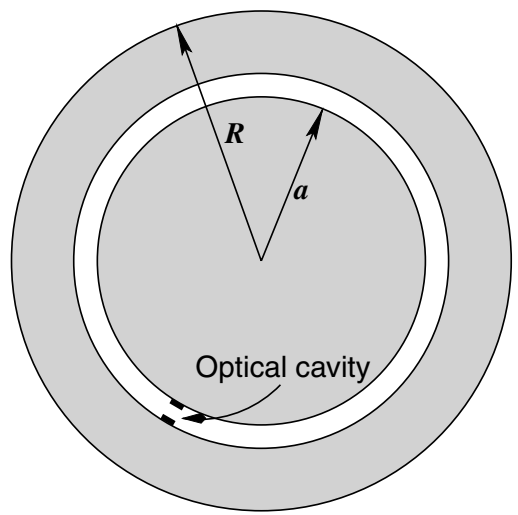

FIG. 1. A dual sphere GW detector with Fabry-Perot cavities as motion sensors.

such loss of response, several resonances from the GWinactive modes appear. While the spectral sensitivity would be still of some interest, we prefer, for brevity, to not discuss it here.

Let us assume that we sense only radial displacements and that the spherical symmetry of the resonators is not broken; suspending a solid spherical resonator has shown to alter only marginally the spectrum of resonances [8]. Using the notation of Refs. [10] and [11], the response to a GW of the solid sphere at its surface and of the hollow at its inner surface (i.e., at the radius $a$ ) are respectively given by expressions of the type

$$
u(\omega)=-\frac{1}{2} \sum_{n=0}^{\infty} b_{n} A_{n 2}(a) \omega^{2} \tilde{h}(\omega) L_{n 2}(\omega),
$$

where $A_{n 2}(a)$ are radial function coefficients, $b_{n}$ are the coefficients in the orthogonal expansion of the response function of each sphere, $L_{n 2}(\omega)$ is the Lorentzian curve associated to the mode $\{n 2\}$, the $n$th quadrupole harmonic, and $\tilde{h}(\omega) \equiv \tilde{h}_{i j}(\omega) n_{i} n_{j}$ is the Fourier amplitude of the GW strain at the sensing point direction, defined by the unit radial vector $\mathbf{n}$ relative to the system's center of mass. Of course, all these quantities must be calculated for either sphere.

Each sensor output is affected by thermal and backaction displacement noise spectral densities, which must be formed for both spheres:

$$
S_{u u}^{[t h+b a]}(\omega)=\sum_{n l} \frac{2 l+1}{4 \pi M}\left|A_{n l}(a)\right|^{2}\left|L_{n l}(\omega)\right|^{2}\left[\frac{2 k T \omega_{n l}^{2}}{Q_{n l} \omega}+\frac{2 l+1}{4 \pi M}\left|A_{n l}(a)\right|^{2} \sum_{j}\left|\mathcal{P}_{l}\left(\mathbf{n} \cdot \mathbf{n}_{j}\right)\right|^{2} S_{F F}^{b a}\right],
$$

where $k$ is Boltzmann's constant, $T$ the sphere's thermodynamic temperature, and $M$ the sphere's mass, whether solid or hollow. $S_{F F}^{b a}$ is the back-action force spectral density, $\mathcal{P}_{l}$ a Legendre polynomial, and $\mathbf{n}_{j}$ the spherical coordinates of the optical cavities $\left(\mathbf{n} \equiv \mathbf{n}_{1}\right)$. The sum over $j$ accounts for the fact that each sensor is additionally affected by the back-action noise forces exerted by the others. The back-action noise force is given by

$$
S_{F F}^{b a}(\omega)=\frac{4}{c^{2} \pi^{2}}(1-\zeta)^{2} h \nu_{l} F^{2} P \frac{1}{1+\left(\frac{2 F L_{c} \omega}{\pi c}\right)^{2}},
$$

where $\nu_{l}$ is the light frequency, $c$ the speed of light, $P$ the light power incident on the cavity, and $\zeta^{2}$ the fraction of light reflected by the cavity at its resonance.

Assuming the noise in the spheres is uncorrelated [18], the total displacement spectral density is the sum of 
expressions similar to Eq. (2) for each sphere, plus a photodetector noise term, the shot noise. The total strain noise spectral density is thus given by

$$
S_{h h}(\omega)=\frac{S_{u u, \text { hollow }}^{[t h+b a]}(\omega)+S_{u u, \text { solid }}^{[t h+b a]}(\omega)+S_{u u}^{\text {shot }}(\omega)}{\left|u_{\text {hollow }}(\omega)-u_{\text {solid }}(\omega)\right|^{2} /|\tilde{h}(\omega)|^{2}} .
$$

Here $S_{u u}^{\text {shot }}(\omega)$ is the photodetector noise which can be written as

$$
S_{u u}^{\text {shot }}(\omega)=4 \times 10^{-33}\left[1+\left(\frac{2 F L_{c} \omega}{\pi c}\right)^{2}\right] \frac{1}{F^{2} P} \frac{\mathrm{m}^{2}}{\mathrm{~Hz}} .
$$

We may now consider the actual GW sensitivity of a system such as this. We take as reference an operation at the Standard Quantum Limit. The SQL is reached at laser powers such that the shot noise and the radiation pressure equally contribute to the total noise and, at the same time, the back-action noise overcomes the mechanical resonator thermal noise. This is the procedure envisioned also for interferometric GW detectors [19]. Note that, since the cavity is short, order of $1 \mathrm{~cm}$, the finesse can be made very high, order of $10^{6}$ and beyond, before losing signal strength, and thus the SQL can be approached at laser powers of the order of $10 \mathrm{~W}$. Moreover, since the bandwidth $\Delta f$ is expected to be wide, $\Delta f \simeq f$, the SQL condition for the mechanical resonators $k T / Q=h \Delta f / 4$ allows $Q / T=10^{8} \mathrm{~K}^{-1}$. Given that one is able to approach the SQL in the readout, then one needs a large cross section to GWs. The resonance frequency fixed, the latter scales as $\rho v_{s}^{5}$, where $\rho$ is the density of the material and $v_{s}$ the velocity of sound.

Molybdenum shows up as an interesting choice, with a sound velocity $v_{s}=6.2 \mathrm{~km} / \mathrm{s}$ and a density $\rho=10 \times$ $10^{3} \mathrm{~kg} / \mathrm{m}^{3}$. The fabrication of the dual sphere may proceed from Mo powders, which can be pressed, sintered to 95\% density, and hot formed to custom shapes. This procedure allows mechanical $Q$ of $2 \times 10^{7}$ at temperatures $\leq 4 \mathrm{~K}$ [20], necessary to approach the SQL as discussed above. The thermal properties of molybdenum are such that no particular difficulties are expected to arise when cooling down such large masses [21]. Building on the experience developed for vibrationally insulating masses of a few tons, as in bar detectors, we are confident that a suitable design can be made for suspending a few tens of tons.

Hot pressed, sintered beryllium is even more interesting, as $v_{s}=13 \mathrm{~km} / \mathrm{s}$ and $\rho=1.9 \times 10^{3} \mathrm{~kg} / \mathrm{m}^{3}$; it has already been used in large sizes (over $1.3 \mathrm{~m}$ ) and its thermal properties make it viable as well. The low-temperature mechanical $Q$ is going to be investigated [22]. Another interesting material is sapphire $\left(v_{s}=10 \mathrm{~km} / \mathrm{s}, \rho=4 \times\right.$ $10^{3} \mathrm{~kg} / \mathrm{m}^{3}$ ), which is already known to show $Q>10^{8}$ at $T<10 \mathrm{~K}$. Sapphire, acting as a substrate of the mirrors, would also minimize thermoelastic effects at low temperatures [23]. Sapphire drawback mainly resides on the diffi- culty of growing large enough crystals and/or joining together several pieces while preserving the high mechanical $Q$. We note that the $\rho v_{s}^{5}$ factor for beryllium is a factor of 2 greater than for sapphire.

A molybdenum detector with $R=0.95 \mathrm{~m}$ and $a=$ $0.57 \mathrm{~m}$, with a small gap in between to place the motion sensing optical cavities, would give an interestingly low strain spectral noise in a rather wide frequency band in the $\mathrm{kHz}$ region (see Fig. 2). Here we plot the SQL spectral strain noise, when the radiation pressure noise is matched to the shot noise at $1.3 \mathrm{kHz}$ : this requires an input light power of $7 \mathrm{~W}$ and $Q / T \geq 2 \times 10^{8} \mathrm{~K}^{-1}$. The spectral strain noise is also shown for a lower light power $P=1 \mathrm{~W}, Q / T=2 \times 10^{7} \mathrm{~K}^{-1}$ possibly more amenable to cryogenic operation at $T \simeq 1 \mathrm{~K}$, but still giving an interesting performance. Note that the spectral sensitivity is contaminated by the thermal noise peaks of the nonquadrupole resonances. The problem of unwanted narrow resonances in the sensitive frequency band is also present in the case of interferometric detectors, and methods have been devised to filter them out $[24,25]$.

Figure 3 shows a comparative plot of the sensitivities of various $\mathrm{GW}$ detectors to come: initial VIRGO [24], the cryogenic interferometer LCGT [26], and LIGO II operated in the narrow band dual-recycled mode [27]. For the proposed dual sphere, we show the SQL operation of the SQL molybdenum system of Fig. 2 with the nonquadrupole resonances suppressed for clarity. The large drop in sensitivity, indicated by the prominent spike towards the right end of the figure, is due to signal cancellation at a frequency $\omega_{\star}$ for which $u_{\text {hollow }}\left(\omega_{\star}\right)=u_{\text {solid }}\left(\omega_{\star}\right)$, which causes the denominator in Eq. (4) to vanish. The presence of such a frequency $\omega_{\star}$ is expected on the basis of the intrinsic structure of Eq. (4), and therefore it must be taken into consideration when one chooses materials

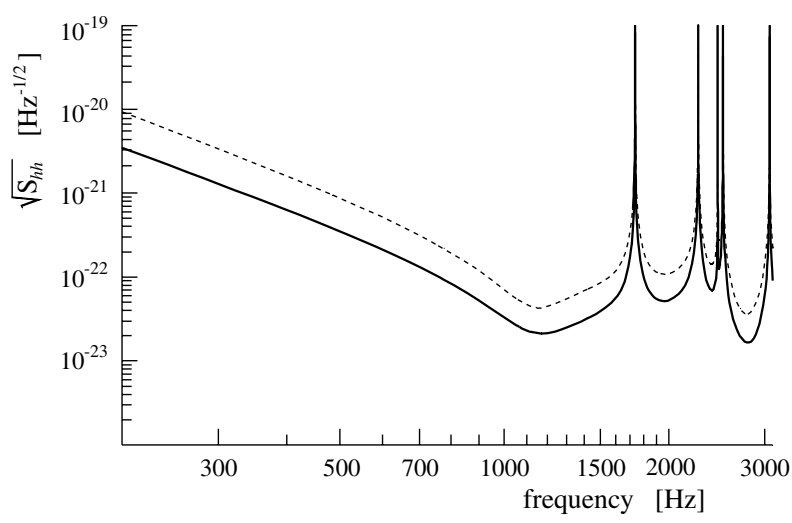

FIG. 2. Spectral strain noise of a molybdenum dual sphere GW detector equipped with $10^{6}$ finesse, $2 \mathrm{~cm}$ long Fabry-Perot cavities as motion sensors. Fundamental mode frequencies: $1100 \mathrm{~Hz}$ for the outer (hollow) sphere, $2825 \mathrm{~Hz}$ for the solid (inner) sphere; total mass: $29+8$ tons. Solid curve: SQL imposed at $1.3 \mathrm{kHz}$, with $P=7 \mathrm{~W}, Q / T \geq 1.6 \times 10^{8} \mathrm{~K}^{-1}$. Dashed curve: $P=1 \mathrm{~W}, Q / T=2 \times 10^{7} \mathrm{~K}^{-1}$. 


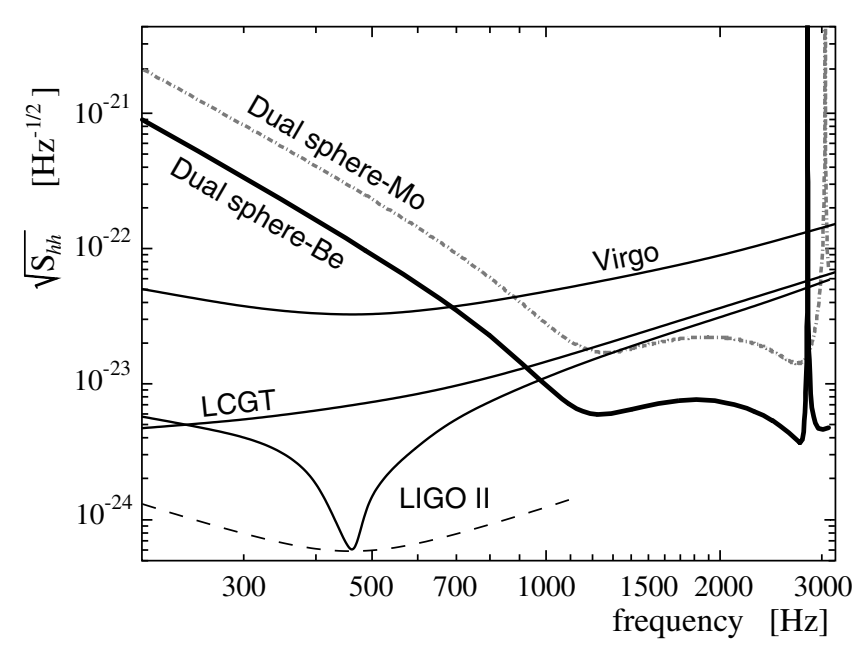

FIG. 3. Spectral strain noise of GW detectors to come compared to dual spheres of molybdenum and beryllium. The LIGO II curve corresponds to the dual-recycled operation tuned to $450 \mathrm{~Hz}$. The dashed line is the location of the minimum for different LIGO II tuning frequencies.

and dimensions. We also show in Fig. 3 the sensitivity of a beryllium system operated at SQL for $1300 \mathrm{~Hz}$, which requires an input light power of $12 \mathrm{~W}$ and $Q / T=2 \times$ $10^{8} \mathrm{~K}^{-1}$; the gain in sensitivity is due to the larger $\rho v_{s}^{5}$ factor. As it can be seen, the dual sphere system well compares with the best foreseen GW detectors, especially in the high frequency region where, e.g., relatively small mass $10 M_{\odot} \mathrm{BH}-\mathrm{BH}$ mergers are expected [28].

In the end, the system we propose may still look similar to a two-mode system in that the most useful band is obtained between the two first quadrupole resonances of the two spheres. However, the concept we propose allows one to choose such two frequencies with a lot of freedom, and in fact to open considerably the band with respect to systems which make use of resonant secondary masses to get the two-mode operation.

We have noted that crucial practical issues for the realization of such a massive system, especially in respect to fabrication, suspensions, and cooling, can be dealt with; however, the level of practicability of the concept may still strongly depend on supportive research.

We thank Stefano Vitale and Michele Bignotto for fruitful discussions. J. A. L. is grateful to INFN Legnaro National Laboratories for hospitality within the EU program "TMR-Access to LSF" (Contract No. ERBFMGECT980110). He also thanks the Spanish MEC for partial support (Contract No. PB96-0384).
*Corresponding author: conti@lnl.infn.it

[1] Z. A. Allen et al., Phys. Rev. Lett. 85, 5046 (2000).

[2] J. C. Price, Phys. Rev. D 36, 3555 (1987).

[3] H. J. Paik, in Proceedings of the 1st E. Amaldi Conference, edited by E. Coccia et al. (World Scientific, Singapore, 1995), p. 201.

[4] J.-P. Richard, Phys. Rev. Lett. 52, 165 (1984).

[5] In the language and notation of Ref. [2], $\Delta f / f=R_{\mathrm{eq}}$, where $R_{\text {eq }}$ is the dimensionless noise resistance. For the readout systems of the past $R_{\text {eq }}=10^{-5} \div 10^{-7}$, while, for a $1 \mathrm{~cm}$-long Fabry-Perot cavity with finesse $F \sim 10^{6}$, input power $P \sim 10 \mathrm{~W}, \lambda=1.064 \mu \mathrm{m}$, it is possible to have $R_{\text {eq }}$ as large as $10^{-1}$.

[6] J.-P. Richard, Phys. Rev. D 46, 2309 (1992).

[7] L. Conti et al., Rev. Sci. Instrum. 69, 554 (1998); L. Conti, M. De Rosa, and F. Marin, Appl. Opt. 39, 5732 (2000).

[8] W. W. Johnson and S. M. Merkowitz, Phys. Rev. Lett. 70, 2367 (1993); S. M. Merkowitz and W. W. Johnson, Phys. Rev. D 56, 7513 (1997), and references therein.

[9] C.Z. Zhou and P. F. Michelson, Phys. Rev. D 51, 2517 (1995).

[10] J. A. Lobo, Phys. Rev. D 52, 591 (1995).

[11] E. Coccia et al., Phys. Rev. D 57, 2051 (1998).

[12] J. A. Lobo, Mon. Not. R. Astron. Soc. 316, 173 (2000).

[13] E. Coccia and V. Fafone, Phys. Lett. A 213, 16 (1996).

[14] M. Cerdonio et al., in Proceedings of the 1st E. Amaldi Conference (Ref. [3]), p. 176.

[15] E. Coccia, J. A. Lobo, and J. A. Ortega, Phys. Rev. D 52, 3735 (1995).

[16] M. Cerdonio et al., Phys. Rev. Lett. 71, 4107 (1993).

[17] S. Vitale et al., Phys. Rev. D 55, 1741 (1997).

[18] Actually, the back-action noise force acting on the cavity mirrors is correlated because the cavity decay time is shorter, or at most comparable, to the inverse of the frequencies of interest. In any case, the correction due to the non-null correlation is estimated to be small and is neglected for simplicity.

[19] W. A. Edelstein, J. Hough, J. R. Pugh, and W. Martin, J. Phys. E Sci. Instrum. 11, 710 (1978).

[20] W. Duffy, Jr. and M. Bassan, Cryogenics 38, 757 (1998).

[21] W. Duffy, Jr., S. Dalal, and M. Quiazon, J. Appl. Phys. 81, 6675 (1997).

[22] G. Frossati (private communication); W. Duffy, Jr. (private communication).

[23] M. Cerdonio, L. Conti, A. Heidmann, and M. Pinard, Phys. Rev. D 63, 082003 (2001).

[24] B. Caron et al., in Proceedings of the 1st E. Amaldi Conference (Ref. [3]), p. 86.

[25] L. S. Finn and S. Mukherjee, Phys. Rev. D 63, 062004 (2001).

[26] K. Kuroda et al., Int. J. Mod. Phys. D 8, 557 (1999).

[27] LIGO document, number M990288-A-M (1999).

[28] E. E. Flanagan and S. A. Hughes, Phys. Rev. D 57, 4535 (1998). 